ARTICLE

DOI: $10.1038 / s 41467-018-04780-x$

\title{
Twinning in metastable high-entropy alloys
}

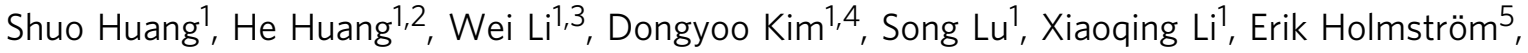 \\ Se Kyun Kwon ${ }^{6}$ \& Levente Vitos (10) 1,3,7
}

Twinning is a fundamental mechanism behind the simultaneous increase of strength and ductility in medium- and high-entropy alloys, but its operation is not yet well understood, which limits their exploitation. Since many high-entropy alloys showing outstanding mechanical properties are actually thermodynamically unstable at ambient and cryogenic conditions, the observed twinning challenges the existing phenomenological and theoretical plasticity models. Here, we adopt a transparent approach based on effective energy barriers in combination with first-principle calculations to shed light on the origin of twinning in highentropy alloys. We demonstrate that twinning can be the primary deformation mode in metastable face-centered cubic alloys with a fraction that surpasses the previously established upper limit. The present advance in plasticity of metals opens opportunities for tailoring the mechanical response in engineering materials by optimizing metastable twinning in high-entropy alloys.

\footnotetext{
${ }^{1}$ Applied Materials Physics, Department of Materials Science and Engineering, Royal Institute of Technology, Stockholm SE-100 44, Sweden. ${ }^{2}$ Science and Technology on Surface Physics and Chemistry Laboratory, Mianyang 621900, PR China. ${ }^{3}$ Department of Physics and Astronomy, Division of Materials Theory, Uppsala University, SE-75120 Uppsala, Sweden. ${ }^{4}$ Department of Physics, Pukyung National University, Busan 608-737, Republic of Korea. ${ }^{5}$ Sandvik Coromant R\&D, 12680 Stockholm, Sweden. ${ }^{6}$ Graduate Institute of Ferrous Technology, Pohang University of Science and Technology, Pohang 37673, Republic of Korea. ${ }^{7}$ Institute for Solid State Physics and Optics, Wigner Research Centre for Physics, H-1525 Budapest, Hungary. Correspondence and requests for materials should be addressed to S.H. (email: shuoh@kth.se) or to S.K.K. (email: sekk@postech.ac.kr) or to L.V. (email: levente@kth.se)
} 
D esigning strong, and at the same time, ductile metals has been among the most ambitious goals for metallurgists. The apparently conflicting mechanisms often resulted in grades compromising between strength and ductility. In spite of the early ideas revolving around $\mathrm{Fe}-\mathrm{Mn}$ alloys ${ }^{1}$, simultaneous improvement of the two properties has been very challenging. The discovery of the advanced high-strength steels near the turn of the century was a pioneering step towards reaching such goals $^{2}$. Recently, the strength-ductility tradeoff was also overcome by designing dual-phase high-entropy alloys (HEAs) ${ }^{3}$, which turned attention towards non-conventional alloys containing multi-principal elements in equal or nearly equal concentrations ${ }^{4,5}$. Successful single-phase solutions also exist such as the fracture-resistant $\mathrm{HEAs}^{6}$ and the exceptional damagetolerance medium-entropy alloys (MEAs) ${ }^{7}$. Further, HEAs with surprising and unanticipated properties are being continuously put forward $^{8-18}$. Parallel with the experimental efforts, computational modeling was also advanced to optimize compositions and reveal the atomic-level mechanisms ${ }^{19}$ behind the strength and ductility improvements.

The common denominator of the most promising routes over the strength-ductility dilemma is the tailored plastic deformation mechanism. Face-centered cubic (fcc) metals normally deform by dislocation glide resulting in good ductility and reduced strength. But in the aforementioned special alloys, the leading deformation modes are twinning and transformation induced plasticity. In particular, twinning was found to play an important role for the enhanced ductility and high strain hardening rate $2,3,6,7,20,21$. There are several phenomenological plasticity models ${ }^{22,23}$ developed to understand the activation of twinning deformation in fcc metals. These models are based on the stacking fault energy (SFE) defined as the excess energy due to the stacking fault connecting the Shockley partial dislocations. For instance, austenitic steels with SFE higher than $10-16 \mathrm{~mJ} \mathrm{~m}^{-2}$ are predicted to show twinning ${ }^{24-26}$, whereas alloys with SFE below this critical value should exhibit deformation-induced phase transformation. Theoretical works also addressed the question of twinnability ${ }^{27-29}$, and they indicate twinning for fcc stable metals with twinning fraction below $50 \%{ }^{27}$.

Within the simplest model, the SFE corresponds to the energy difference between the hexagonal close-packed (hcp) and fcc structures ${ }^{30}$. Thus, the sizable positive SFE required for twinning by the phenomenological models ${ }^{22,23}$ implies a thermodynamically stable fcc phase. Traditional alloys like austenitic steels ${ }^{31}$ belong to this group. Since the discovery of the first fcc HEAs based on $3 \mathrm{~d}$ transition metals ${ }^{4,5}$, it was expected that these multi-component systems also follow a similar plastic deformation scenario. However, recent experimental works reported a pressure-induced fcc-hcp transition in $\mathrm{CrMnFeCoNi}$, and the hcp phase was retained after decompression to ambient pressure ${ }^{32,33}$. The observed equilibrium phase boundary between the fcc and hcp phases was placed near $600 \mathrm{~K}^{33}$. For the multicomponent alloys in question $3,6,7,20,34-37$, including CrMnFe$\mathrm{CoNi}$, ab initio theory fully confirms the experimental findings by predicting a thermodynamically unstable fcc lattice at low temperature. Hence, both experiment and theory suggest that the metastable fcc CrMnFeCoNi alloy should possess very small positive or negative SFEs at ambient and cryogenic conditions. But such SFE values in connection with the phenomenological models $^{22,23}$ clearly fail to explain the observed twinning 6 . Thus, the available experimental and theoretical evidences severely challenge the validity of the widely-used SFE-based plasticity models in the case of metastable HEAs ${ }^{22,27}$. Although this fundamental problem was recognized by several groups ${ }^{19,34-38}$, so far no unified solution has been put forward.
Here, we employ first-principle quantum mechanical tools to resolve the twinning mechanism in metastable multi-component alloys. We select the representative $\mathrm{Fe}_{80-x} \mathrm{Mn}_{x} \mathrm{Co}_{10} \mathrm{Cr}_{10}(30 \leq x \leq$ 45) and CrMnFeCoNi HEAs ${ }^{3,6}$, and $\mathrm{CrCoNi} \mathrm{MEA}^{7}$, for which reliable experimental data for the deformation mechanisms exist. Based on the intrinsic energy barriers, we show that it is the particular chemistry that is primarily responsible for the emergence of twinning in metastable alloys, and decreasing temperature efficiently drives the system across various deformation modes. The inherent affine shear strain prior to slip, which is associated to the relatively large critical twinning stress characteristic to the present systems, further promotes twinning. Our results are expected to help optimizing the properties of engineering materials involving HEAs towards excellent strength-ductility combinations.

\section{Results}

Deformation modes. The leading deformation modes in fcc metals are stacking fault (SF), twinning (TW), and fullslip (SL). The active mode is decided by the competition between the corresponding effective energy barriers (EEBs): $\bar{\gamma}_{\mathrm{SF}}(\theta)=\gamma_{\mathrm{usf}} / \cos \theta, \bar{\gamma}_{\mathrm{TW}}(\theta)=\left(\gamma_{\mathrm{utf}}-\gamma_{\mathrm{isf}}\right) / \cos \theta$, and $\bar{\gamma}_{\mathrm{SL}}(\theta)=$ $\left(\gamma_{\text {usf }}-\gamma_{\text {isf }}\right) / \cos \left(60^{\circ}-\theta\right)$ for SF, TW, and SL, respectively ${ }^{27}$. In these expressions, $\theta$ varies between $0^{\circ}$ and $60^{\circ}$ and reflects the effect of grain orientation relative to the resolved shear direction, $\gamma_{\text {isf }}$ is the intrinsic SFE, $\gamma_{\text {usf }}$ the unstable SFE, and $\gamma_{\text {utf }}$ the unstable twin fault energy. These intrinsic material parameters (IMPs) describe the generalized SFE $(\gamma \text {-surface })^{39}$ and are determined from a series of ab initio calculations. According to the above EEBs, twinning is possible for $\bar{\gamma}_{\mathrm{TW}}(\theta) \leq \bar{\gamma}_{\mathrm{SF}}(\theta)$, otherwise martensitic transformation occurs. Furthermore, due to the shear direction dependence, SF and SL or TW and SL can coexist in a homogenous material, but SF and TW are exclusive to each other independently of $\theta$ (they possess similar Schmid factors). We mention that SF and TW can coexist in special alloys (e.g., interstitial HEAs ${ }^{16-18}$ ) due to the broadening of their boundary. For the sake of simplicity, in the following we do not always mention SL when discussing the SF and TW mechanisms.

Using the calculated EEBs, first we explore the deformation modes of the $\mathrm{Fe}_{80-x} \mathrm{Mn}_{x} \mathrm{Co}_{10} \mathrm{Cr}_{10}$ alloys as a function of temperature and composition. These alloys are reported to exhibit twinning or martensitic transformation depending on $\mathrm{Mn}$ content $^{3}$. The room-temperature $\left(\bar{\gamma}_{\mathrm{SF}}-\bar{\gamma}_{\mathrm{TW}}\right) \equiv \bar{\gamma}_{\mathrm{SF}}\left(0^{\circ}\right)-$ $\bar{\gamma}_{\mathrm{TW}}\left(0^{\circ}\right)$ values are plotted in Fig. 1 as a function of Mn content. We find that $\left(\bar{\gamma}_{\mathrm{SF}}-\bar{\gamma}_{\mathrm{TW}}\right)$ changes sign with increasing $\mathrm{Mn}$ concentration. Namely, for $x \geq 33$, TW is favored against SF, whereas for $x<33 \mathrm{SF}$ is activated. In addition, SL is present in both regimes with $\theta$ increasing towards $60^{\circ}$ (not shown). These theoretical predictions are in perfect agreement with observations $^{3}$ and confirm that the present theory correctly captures the observed trends of the deformation modes as a function of composition.

Figure 1 also shows the free energy difference $\Delta F \equiv\left(F_{\text {hcp }}-\right.$ $\left.F_{\text {fcc }}\right)$ for $\mathrm{Fe}_{80-x} \mathrm{Mn}_{x} \mathrm{Co}_{10} \mathrm{Cr}_{10}$ by varying $x$. Negative $\Delta F$ indicates a thermodynamically unstable fcc lattice, which turns out to be the case for all compositions considered here. By combining the behavior of $\Delta F$ with that of $\left(\bar{\gamma}_{\mathrm{SF}}-\bar{\gamma}_{\mathrm{TW}}\right)$ in terms of Mn content, we arrive to a very interesting and unprecedented phenomenon. Namely, in $\mathrm{Fe}_{80-x} \mathrm{Mn}_{x} \mathrm{Co}_{10} \mathrm{Cr}_{10}$ HEAs, the room-temperature twinning is associated with a metastable fcc phase for $33 \leq x \leq 50$ (upper limit is estimated by extrapolating the slope of $\Delta F$ towards high $\mathrm{Mn}$ content). We emphasize that since these alloys possess negative or very small positive $\gamma_{\text {isf }}$ (as shown below), the empirical plasticity models based on $\mathrm{SFE}^{22,23}$ would dictate SF rather than TW mechanism, opposed to the present theoretical 
and former experimental findings ${ }^{3}$. Actually, in these special alloys, $\bar{\gamma}_{\mathrm{TW}}$ is lower than $\bar{\gamma}_{\mathrm{SF}}$, which induces twins instead of stacking faults toward martensitic transformation, despite of the negative $\Delta F$. Below, we refer to this phenomenon as metastable twinning (MTW).

Next, we consider the effect of temperature on the deformation modes. We recall that tuning the deformation mechanisms by temperature is a well-known routine to alter the plasticity of materials ${ }^{6,7}$. Figure 2a (solid symbols) displays $\left(\bar{\gamma}_{\mathrm{SF}}-\bar{\gamma}_{\mathrm{TW}}\right)$ and $\Delta F$ as a function of temperature for the $\mathrm{Fe}_{40} \mathrm{Mn}_{40} \mathrm{Co}_{10} \mathrm{Cr}_{10}$ HEA. Alloys with different Mn-levels follow qualitatively the same behavior and thus they are not included in the figure. At high temperature, the system is in the positive $\Delta F$ and TW $+\mathrm{SL}$ regime. Similar normal behavior is observed at very low temperature, where negative $\Delta F$ is associated with $\mathrm{SF}+\mathrm{SL}$. However, there exists a temperature interval where the $\mathrm{Fe}_{80-x} \mathrm{Mn}_{x} \mathrm{Co}_{10} \mathrm{Cr}_{10}$ HEAs behave unexpectedly: TW is activated in spite of the negative $\Delta F$. For $x=35,40$, and 45 , the abnormal

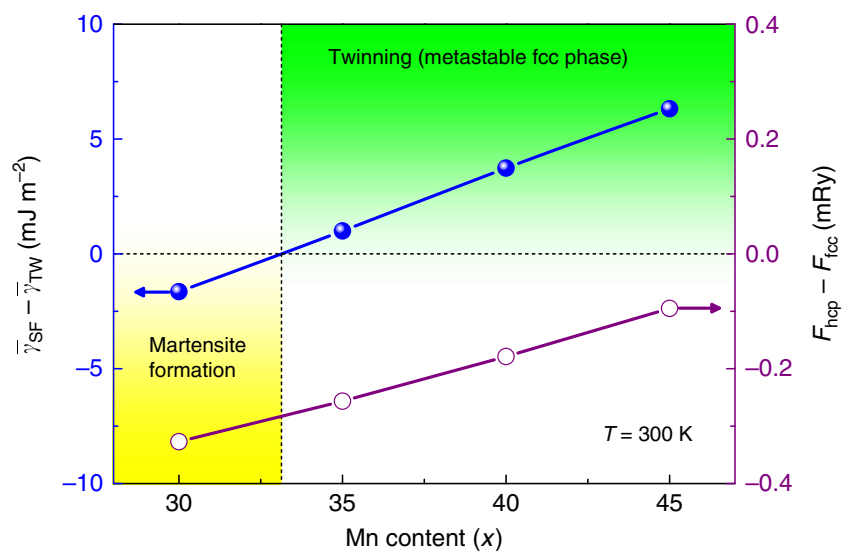

Fig. 1 Composition-dependent effective energy barrier difference $\left(\bar{\gamma}_{\mathrm{SF}}-\bar{\gamma}_{\mathrm{TW}}\right)$ for the $\mathrm{Fe}_{80-x} \mathrm{Mn}_{x} \mathrm{CO}_{10} \mathrm{Cr}_{10}$ alloys at room-temperature. Negative $\left(\bar{\gamma}_{\mathrm{SF}}-\bar{\gamma}_{\mathrm{TW}}\right)$ indicates hcp martensite formation (yellow area), while positive one implies twinning (green area). Open circles denote the structural energy difference $\left(F_{\mathrm{hcp}}-F_{\mathrm{fcc}}\right)$, where $F_{\mathrm{hcp}}$ and $F_{\mathrm{fcc}}$ are the free energies of hcp and fcc structures, respectively interval includes room-temperature, whereas for $x=30$ it is shifted above room-temperature. Similar calculated $\left(\bar{\gamma}_{\mathrm{SF}}-\bar{\gamma}_{\mathrm{TW}}\right)$ and $\Delta F$ versus temperature data for $\mathrm{CrMnFeCoNi}$ and $\mathrm{CrCoNi}$ alloys are shown in Fig. 2b,c (solid symbols), respectively. According to the free energy differences, for these two alloys, the hcp structure is more stable than the fcc one at low temperatures. Our findings regarding the structural stability are fully consistent with the previous theoretical works ${ }^{34-36,40,41}$ (see also Supplementary Note 1 and Supplementary Figs. 1 and 2). When comparing the trends for $\left(\bar{\gamma}_{\mathrm{SF}}-\bar{\gamma}_{\mathrm{TW}}\right)$ and $\Delta F$, it turns out that the peculiar MTW appears in the $\mathrm{CrMnFeCoNi}$ alloy between $\sim 300$ and $\sim 460 \mathrm{~K}$, and the metastable $\mathrm{CrCoNi}$ alloy shows no twinning in the temperature range considered here. Before contrasting these theoretical predictions with the experimental observations, we investigate the calculated generalized SFEs.

Generalized stacking fault energy. The room-temperature $\gamma$ surfaces of $\mathrm{Fe}_{40} \mathrm{Mn}_{40} \mathrm{Co}_{10} \mathrm{Cr}_{10}$, CrMnFeCoNi, and CrCoNi alloys are shown in Fig. 3a. Remarkably, all three alloys possess $\gamma_{\text {usf }} \approx$ $\gamma_{\text {utf }}$ with small (or negative) $\gamma_{\text {isf }}$. Similar IMPs were reported by several former theoretical woks ${ }^{34,36}$ (see also Supplementary Fig. 1). This particular shape of the $\gamma$-surface differs from that of conventional fcc alloys ${ }^{27,31,42}$ and has important implication on the deformation mechanism as explained in the following paragraphs. The $\gamma$-surfaces in Fig. 3a were obtained from alias shear (inset in Fig. 3a), i.e., by shifting the upper part of the fcc $\{111\}$ layers along the $\langle 11 \overline{2}\rangle$ direction. In practice, this process requires a critical resolved shear stress, which raises a small but finite affine shear: all atomic planes are actually elastically strained along the shear direction (inset in Fig. 3b ${ }^{43}$. The affine shear strain-dependence of $\left(\bar{\gamma}_{\mathrm{SF}}-\bar{\gamma}_{\mathrm{TW}}\right)$ is presented in Fig. 3b. Interestingly, increasing affine shear strain increases $\left(\bar{\gamma}_{\mathrm{SF}}-\bar{\gamma}_{\mathrm{TW}}\right)$ and thus favors twin nucleation for all alloys considered here. In particular, MTW is predicted in CrCoNi alloys as well with increasing affine shear strain. Therefore, in addition to the intrinsic materials properties, the strain conditions also sensitively affect the twinnability of these alloys. The sensitivity of the deformation mode to the shearing conditions should be attributed to the special shape of the $\gamma$-surfaces in Fig. 3a. We note that the beneficial effect of strain on the twinnability is mentioned in connection with the empirical deformation maps as well ${ }^{22}$.
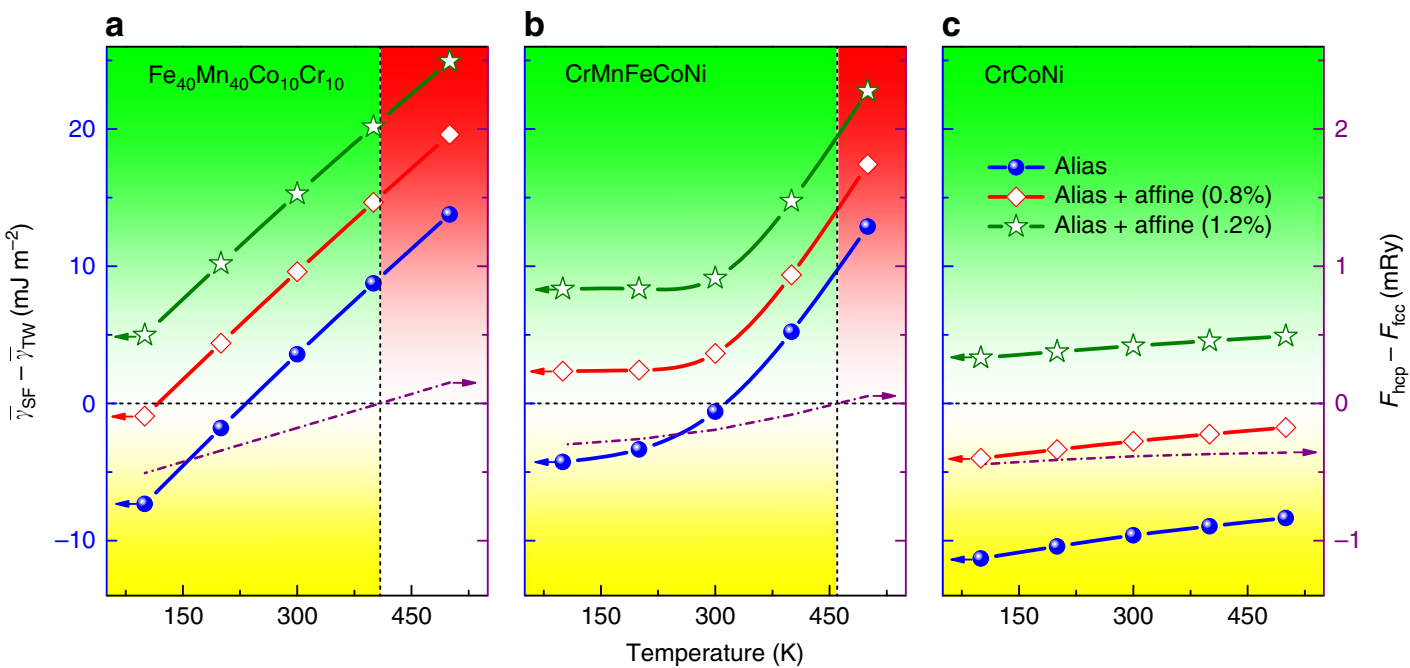

Fig. 2 Temperature-dependent effective energy barrier difference $\left(\bar{\gamma}_{\mathrm{SF}}-\bar{\gamma}_{\mathrm{TW}}\right)$. a-c show the results for $\mathrm{Fe}_{40} \mathrm{Mn}_{40} \mathrm{Co}_{10} \mathrm{Cr}_{10}, \mathrm{CrMnFeCoNi}$ and $\mathrm{CrCoNi}_{\mathrm{C}}$ alloys respectively. The structural energy differences $\left(F_{\mathrm{hcp}}-F_{\mathrm{fcc}}\right)$ are plotted by dash-dot lines for comparison. Solid lines with symbols denote $\left(\bar{\gamma}_{\mathrm{SF}}-\bar{\gamma}_{\mathrm{TW}}\right)$ without (alias, blue filled symbols) and with $0.8 \%$ and $1.2 \%$ affine shear strains (open symbols), respectively. The metastable twinning regime (MTW) is marked by the green, the martensite formation regime (SF) by the yellow, and the normal twinning regime (TW) by the red areas 


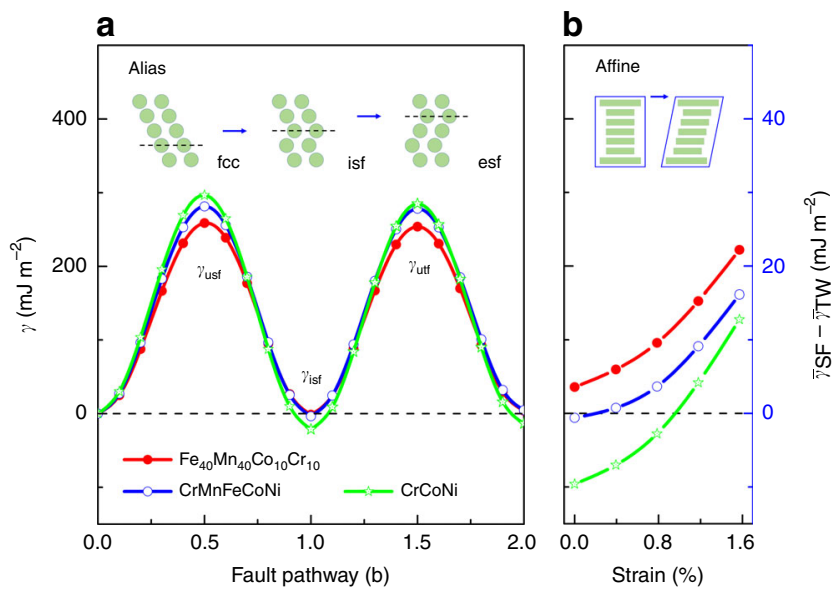

Fig. 3 Generalized stacking fault energy curves for the $\mathrm{Fe}_{40} \mathrm{Mn}_{40} \mathrm{Co}_{10} \mathrm{Cr}_{10}$, $\mathrm{CrMnFeCoNi}$, and $\mathrm{CrCoNi}$ alloys at room-temperature. In panel $\mathbf{a}, \mathbf{b}=$ $\frac{1}{6}\langle 11 \overline{2}\rangle$ is the Burgers vector of the partial dislocation (in units of fcc lattice parameter). $\gamma_{\text {isf }}$, intrinsic stacking fault energy; $\gamma_{\text {usf }}$, unstable stacking fault energy; $\gamma_{\text {utf }}$ unstable twining fault energy. Panel $\mathbf{b}$ shows $\left(\bar{\gamma}_{\mathrm{SF}}-\bar{\gamma}_{\mathrm{TW}}\right)$ as a function of the affine shear strain

For CrMnFeCoNi alloy, the measured critical twinning stress is around $235 \pm 10 \mathrm{MPa}^{44}$. Combining this value with the resolved shear modulus $(48 \mathrm{GPa})$, for the affine shear strain we obtain $\sim 0.8 \%$ as the characteristic value ${ }^{45}$. The $\mathrm{Fe}_{40} \mathrm{Mn}_{40} \mathrm{Co}_{10} \mathrm{Cr}_{10}$ and CrCoNi alloys are expected to possess slightly smaller and larger strains, respectively, as suggested by their EEBs (Fig. 3a). The impact of the strain on the temperature-dependent $\left(\bar{\gamma}_{\mathrm{SF}}-\bar{\gamma}_{\mathrm{TW}}\right)$ for the considered alloys is illustrated in Fig. 2 for 0.8 and $1.2 \%$ affine shear strains (open symbols). In the sheared $\mathrm{Fe}_{40} \mathrm{Mn}_{40^{-}}$ $\mathrm{Co}_{10} \mathrm{Cr}_{10}$, transformation-induced plasticity happens only at very low temperature, otherwise MTW or regular TW is present. In $\mathrm{CrCoNi}$, about $0.9 \%$ affine shear strain produces MTW at all temperatures, which is in line with the recent experiments ${ }^{7}$. In the sheared CrMnFeCoNi (strains $\gtrsim 0.7 \%$ ), MTW occurs for all temperatures below $\sim 460 \mathrm{~K}$, which explains the reported stress-strain behavior at cryogenic condition ${ }^{6}$. On the other hand, for CrMnFeCoNi, the calculation without shear strain predicts a martensitic transformation below $\sim 300 \mathrm{~K}$, which is consistent with the pressure-induced fcc-hcp phase transition ${ }^{32,33}$.

Mechanism of metastable twinning. So far we defined MTW as twinning deformation when $\Delta F \leq 0$. Below, we reformulate the MTW condition using the SFE and related IMPs. An intrinsic stacking fault in an fcc lattice can be treated as a two-layer embryo with the hcp structure embedded in the fcc matrix. Accordingly, the SFE is often expressed as ${ }^{46} \gamma_{\text {isf }}=2 \Delta F / A+2 \sigma^{*}$, where $A$ is the area of the stacking fault and $\sigma^{*}$ is the pseudointerfacial energy between the hcp embryo and the fcc matrix. Hence, $\gamma_{\text {isf }} \leq 2 \sigma^{*}$ can be taken as the condition for the thermodynamic stability of the hcp lattice. Using our calculated $\gamma_{\text {isf }}$ and $\Delta F$ values, for the present alloys we obtain $\sigma^{*} \approx 4-9 \mathrm{~mJ} \mathrm{~m}^{-2}$, which compares well with $8-9 \mathrm{~mJ} \mathrm{~m}^{-2}$ reported for $\mathrm{Fe}-\mathrm{Cr}-\mathrm{Ni}$ ' alloys encompassing about $14-20 \% \mathrm{Cr}$ and $16-20 \% \mathrm{Ni}^{47}$. In applications, $\sigma^{*}$ is often considered to be independent of temperature $^{46,48}$.

In terms of IMPs, twinning can occur if $\gamma_{\text {isf }}>\gamma_{\text {utf }}-\gamma_{\text {usf }}$. Elemental fcc metals and austenitic steels ${ }^{31,39,42,49}$ have $\gamma_{\text {utf }}>\gamma_{\text {usf }}$, and thus a positive SFE is required to activate TW. The opposite case $\gamma_{\text {utf }}<\gamma_{\text {usf }}$ is characteristic to hcp metals ${ }^{27,50}$, for which however the large negative $\gamma_{\text {isf }}$ forbids TW. These two cases provide the physical background for the phenomenological

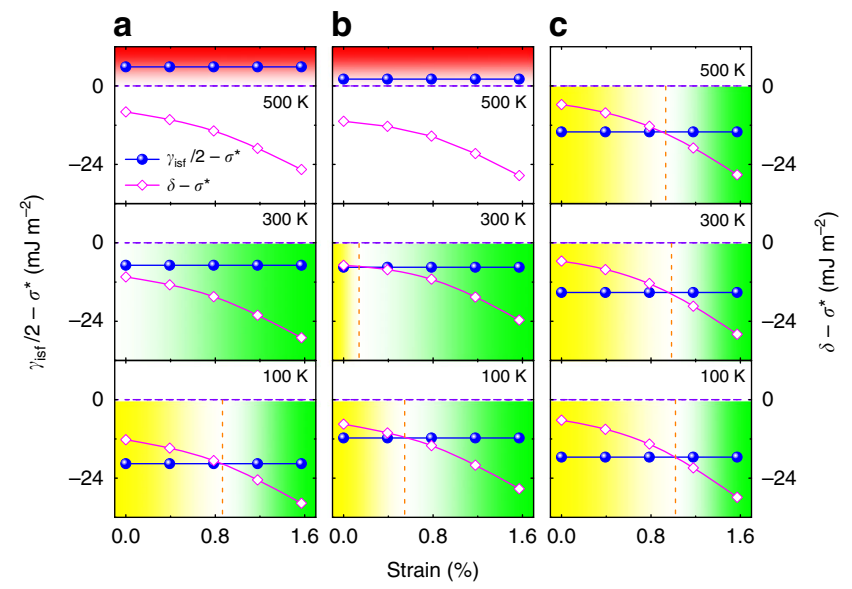

Fig. 4 Twinning parameters as a function of the affine shear strain. a-c show the strain-dependence of $\left(\gamma_{\text {isf }} / 2-\sigma^{*}\right)$ and $\left(\delta-\sigma^{*}\right)$ for three different temperatures for the $\mathrm{Fe}_{40} \mathrm{Mn}_{40} \mathrm{Co}_{10} \mathrm{Cr}_{10}, \mathrm{CrMnFeCoNi}$, and $\mathrm{CrCoNi}$ alloys, respectively. Metastable twinning corresponds to $\left(\gamma_{\text {isf }} / 2-\right.$ $\left.\sigma^{*}\right)>\left(\delta-\sigma^{*}\right)$ and $\left(\gamma_{\text {isf }} / 2-\sigma^{*}\right)<0$ (green area), martensitic transformation to $\left(\gamma_{\text {isf }} / 2-\sigma^{*}\right)<\left(\delta-\sigma^{*}\right)$ (yellow area), and normal twinning to $\left(\gamma_{\text {isf }} / 2-\right.$ $\left.\sigma^{*}\right)>0$ (red area). The latter occurs in $\mathrm{Fe}_{40} \mathrm{Mn}_{40} \mathrm{Co}_{10} \mathrm{Cr}_{10}$ and $\mathrm{CrMnFeCoNi}$ alloys at high temperature but not in $\mathrm{CrCoNi}$. Metastable twinning is present in all three alloys within certain strain-temperature windows

correlations between SFE and the deformation modes ${ }^{22}$. However, twinning can take place also when $\gamma_{\text {utf }} \lesssim \gamma_{\text {usf }}$ if $\gamma_{\text {isf }}$ remains larger than a small (negative) critical value. Numerically, for twinning one should have $\gamma_{\text {isf }}>2 \delta$ where $\delta \equiv \gamma_{\text {utf }}-\gamma_{\text {usf }}-\gamma_{\text {isf }} / 2$ expresses the deviation relative to the so-called universal scaling law ${ }^{51}$. Theoretical calculations show that for fcc metals and alloys, $\delta$ can be both positive and negative ${ }^{42}$, and its absolute value is usually around a few $\mathrm{mJ} \mathrm{m}^{-2}$. Combining this constraint for twinning with the one for the lattice instability, for the condition of MTW, we obtain $\delta<\gamma_{\text {isf }} / 2<\sigma^{*}$. Hence, MTW can only be realized in systems where $\delta$ is below the pseudointerfacial energy.

The above condition excludes from the MTW family, all metals and alloys, which possess $\gamma_{\text {isf }}>2 \sigma^{*}$ at all relevant temperatures. But for the present multi-component alloys ${ }^{38}$, as well as for the paramagnetic $\mathrm{Fe}^{52}$ or the low-Ni austenitic stainless steels ${ }^{31}, \gamma_{\text {isf }}$ is negative at static conditions and increases with increasing temperature. In such systems, $\gamma_{\text {isf }} / 2$ usually scans the interval between $\delta$ and $\sigma^{*}$ when changing the temperature, and thus the MTW mechanism might become effective within a specific temperature range. The sign of $\left(\delta-\sigma^{*}\right)$ in turn is decided by the chemistry and its magnitude is sensitive to the affine shear strain preceding the deformation. Since $\sigma^{*}$ is usually small (typically below $10 \mathrm{~mJ} \mathrm{~m}^{-2}$ ), mostly alloys with very small or negative $\delta$ are expected to enter the MTW regime upon changing the temperature. Alloys with positive $\delta$ (but still below $\sigma^{*}$ ), such as $\mathrm{Fe}-\mathrm{Cr}-\mathrm{Ni}^{31}$, are predicted to have a very narrow temperature interval for MTW. Finally, alloys with $\delta>\sigma^{*}$ transform directly from the normal TW region of the stable austenitic phase to the SF region with lowering the temperature.

The two critical parameters for MTW, $\left(\gamma_{\text {isf }} / 2-\sigma^{*}\right)$ and $\left(\delta-\sigma^{*}\right)$, are shown in Fig. 4 for $\mathrm{Fe}_{40} \mathrm{Mn}_{40} \mathrm{Co}_{10} \mathrm{Cr}_{10}, \mathrm{CrMnFe}-$ $\mathrm{CoNi}$, and $\mathrm{CrCoNi}$ as a function of affine shear strain and temperature. It is remarkable that all three alloys satisfy the $\left(\delta-\sigma^{*}\right)<0$ condition at all temperatures and strains from the figure and therefore they are prone to MTW. At the same time, the calculated relative SFEs $\left(\gamma_{\text {isf }} / 2-\sigma^{*}\right)$ increase with temperature but remain negative below $410 \mathrm{~K}$ for $\mathrm{Fe}_{40} \mathrm{Mn}_{40} \mathrm{Co}_{10} \mathrm{Cr}_{10}, 460$ 
$\mathrm{K}$ for $\mathrm{CrMnFeCoNi}$, and at all temperatures considered here in the case of CrCoNi. Hence, MTW should appear within certain temperature and strain intervals in all three multi-component alloys. These findings are in perfect line with the present theoretical predictions in Fig. 2 as well as with the available observations $3,6,7,20$.

Stacking fault energy and metastable twinning. In order to express the deformation modes in terms of the experimentally accessible SFE, in Fig. 5a we plot the relationship between $\left(\bar{\gamma}_{\mathrm{SF}}-\bar{\gamma}_{\mathrm{TW}}\right)$ and $\gamma_{\text {isf }}$ for $\mathrm{Fe}_{40} \mathrm{Mn}_{40} \mathrm{Co}_{10} \mathrm{Cr}_{10}$ for temperatures between 100 and $500 \mathrm{~K}$ and three affine strain levels. In the stressfree case, MTW is present for $\gamma_{\text {isf }}$ between -8 and $10 \mathrm{~mJ} \mathrm{~m}^{-2}$, i.e., both negative and small positive SFE values are associated with TW. Increasing the affine shear strain, the maximum allowed $\gamma_{\text {isf }}$ for MTW is shifted slightly towards positive values due to the weak stress-induced increase of $\sigma^{*}$. Furthermore, the lowest SFE for which twinning is observed decreases strongly with strain and thus broadening the range for MTW. For $0.8 \%$ strain, which is close to the estimated value for $\mathrm{CrMnFeCoNi}$, the lowest SFE where TW is the active deformation mode (in addition to SL) is $-16.5 \mathrm{~mJ} \mathrm{~m}^{-2}$. The room-temperature SFE values for strains up to $0.8 \%$ vary between -1.3 and $2.3 \mathrm{~mJ} \mathrm{~m}^{-2}$, and lie well inside the MTW region. These SFEs are far below the empirical limit for twinning $\left(10-16 \mathrm{~mJ} \mathrm{~m}^{-2}\right)$ and confirm that classical SFE-based deformation models ${ }^{22,23}$ fail to account the observed twinning in this $\mathrm{HEA}^{3}$. In Fig. 5b, we propose a schematic deformation mode diagram based on the theoretical data obtained for $\mathrm{Fe}_{40} \mathrm{Mn}_{40} \mathrm{Co}_{10} \mathrm{Cr}_{10}$. We mention that the deformation maps for $\mathrm{CrMnFeCoNi}$ and $\mathrm{CrCoNi}$ (not shown) are qualitatively the same as that in Fig. 5b, but the boundaries between SF and MTW are slightly shifted towards larger SFE values. Like in the phenomenological diagram ${ }^{22}$, in Fig. $5 \mathrm{~b}$, the SF mode occurs for negative and the TW mode for positive SFE. Interestingly, the present theoretical SFE values for normal TW are above $7-14 \mathrm{~mJ} \mathrm{~m}^{-2}$ (depending on the affine shear level), which agree well with 6-12 $\mathrm{mJ} \mathrm{m}{ }^{-2}$ obtained by correcting the empirical critical values of twinning ${ }^{24-26}$ by the estimated strain contribution near the partial dislocations ${ }^{53}$. However, in the deformation mode map for MEAs and HEAs, there is a broad MTW region, which broadens towards both positive and negative SFE values with increasing affine strain. In contrast, in the case of traditional alloys like austenitic stainless steels, the MTW region is very narrow or completely missing.

The present theoretical exploration opens opportunities to fine tune the plastic flow mechanisms in HEAs. One important example is the enhanced twinning probability $\left(f_{\mathrm{TW}}\right)$, which means that an increased number of randomly oriented grains exhibit twinning. In ideal metals $(\delta=0)$, we obtain $f_{\mathrm{TW}}=50 \%$ for $\gamma_{\text {isf }}=0 \mathrm{~mJ} \mathrm{~m}^{-2}$, meanwhile in metals with $\delta>0$, the twinning fraction turns below $50 \%$. For instance, for $\mathrm{Fe}_{68} \mathrm{Cr}_{20} \mathrm{Ni}_{12}$ alloy ${ }^{31}$, $\delta=7 \mathrm{~mJ} \mathrm{~m}^{-2}$ and $\gamma_{\text {usf }}=276 \mathrm{~mJ} \mathrm{~m}^{-2}$ give $f_{\mathrm{TW}}=45 \%$. However, in alloys exhibiting MTW, $f_{\mathrm{TW}}$ can reach values substantially higher than the previously established upper limit. For instance, the present results for $\mathrm{Fe}_{40} \mathrm{Mn}_{40} \mathrm{Co}_{10} \mathrm{Cr}_{10}$ yield $\delta=-4 \mathrm{~mJ} \mathrm{~m}{ }^{-2}$ (neglecting the affine strain) and thus $f_{\mathrm{TW}}=53 \%$. Hence the TW fraction for the present HEA is $\sim 18 \%$ larger than the value obtained for steels.

We have investigated the plastic deformation in multicomponent alloys and presented a transparent atomic-level theory for the MTW mechanism. The theory is based on the EEBs derived from first-principle calculations. We focus on the high strength and high ductility alloys, $\mathrm{Fe}_{80-x} \mathrm{Mn}_{x} \mathrm{Co}_{10} \mathrm{Cr}_{10}$, $\mathrm{CrMnFeCoNi}$, and $\mathrm{CrCoNi}$, in which twinning was reported to be a fundamental deformation mechanism. Both theoretical and

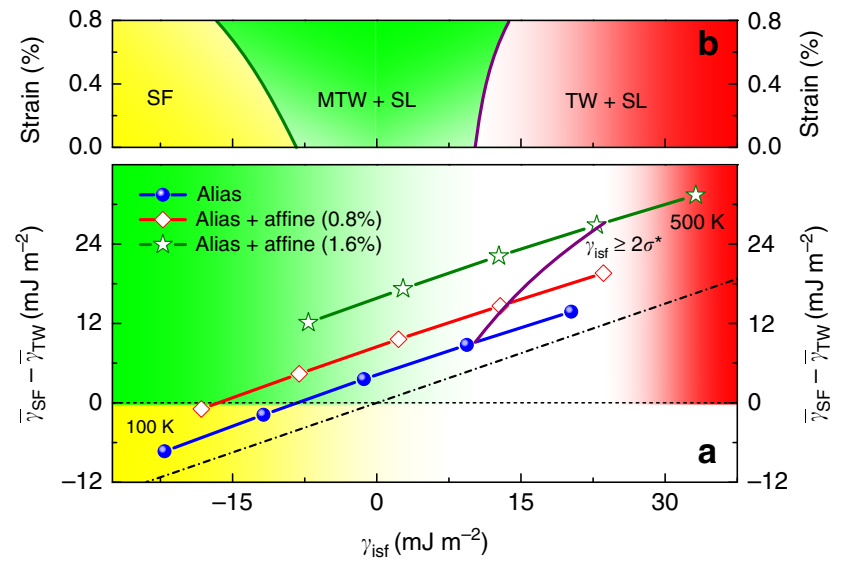

Fig. 5 Deformation modes for the $\mathrm{Fe}_{40} \mathrm{Mn}_{40} \mathrm{Co}_{10} \mathrm{Cr}_{10}$ alloy as a function of intrinsic stacking fault energy $\left(\gamma_{\text {isf }}\right)$. Panel a shows the effective energy barrier difference for temperatures between 100 and $500 \mathrm{~K}$, and for three different affine shear strains. The dash-dot lines marks the ideal relation $\gamma_{\text {uff }}=\gamma_{\text {usf }}+\gamma_{\text {isf }} / 2$ (i.e., the $\delta=0$ case). The solid line is the approximate boundary between metastable twinning (green area) and normal twinning (red area) regions in terms of $\gamma_{\text {isf }}$. Martensitic transformation (yellow area) occurs for $\gamma_{\text {isf }}<-8.4 \mathrm{~mJ} \mathrm{~m}^{-2}$ for the strain-free case, and this boundary is shifted towards lower values with increasing affine strain. The schematic deformation mode diagram shown in panel $\mathbf{b}$ is based on the theoretical data obtained for $\mathrm{Fe}_{40} \mathrm{Mn}_{40} \mathrm{Co}_{10} \mathrm{Cr}_{10}$

experimental results suggest that the studied alloys possess a thermodynamically unstable fcc lattice at ambient and cryogenic conditions, which questions the phenomenological plasticity models. In contrast to those models, the present theory demonstrates that twinning can be the primary deformation mode for metastable fcc alloys with a twinning fraction that surpasses the previously established upper limit. It is found that small affine shear strain can further amplify the MTW phenomenon. The disclosed behavior of the present MEAs and HEAs calls for a critical revision of the previously adopted design criteria regarding twinning-induced plasticity. Our quantum theory aided understanding of the plasticity shows that a precise control of the deformation mechanisms is feasible in these new families of multi-component alloys, which can ultimately facilitate the optimal harvesting of their properties.

\section{Methods}

Theoretical methodology. The present calculations were based on densityfunctional theory formulated within the generalized gradient approximation ${ }^{54}$ and the exact muffin-tin orbitals formalism ${ }^{55}$. The Kohn-Sham equations were solved within the scalar-relativistic and soft-core schemes. Since the Curie temperatures estimated via the mean field approximation ${ }^{56}$ are below $77 \mathrm{~K}$, all alloys were described in the paramagnetic state modeled by the disordered local magnetic moment approximation ${ }^{57}$. The chemical and magnetic disorders were taken into account using the coherent potential approximation ${ }^{55}$. The free energies included the lattice expansion and magnetic entropy terms. For a randomly oriented polycrystalline alloy, the fraction of grains showing TW was estimated from the IMPs via ${ }^{27} f_{\text {TW }}=\frac{1}{60^{\circ}} \tan ^{-1}\left[\frac{\sqrt{3}}{3}\left(1-\frac{4 \delta}{\gamma_{\text {uff }}}\right)\right]$.

Data availability. The data that support the findings of this study are available from the corresponding authors upon reasonable request.

Received: 8 March 2017 Accepted: 23 May 2018

Published online: 18 June 2018

\section{References}

1. Hadfield, R. A. Hadfield's manganese steel. Science 12, 284-286 (1888). 
2. Grässel, O., Krüger, L., Frommeyer, G. \& Meyer, L. W. High strength Fe-Mn(Al, Si) TRIP/TWIP steels development-properties-application. Int. J. Plast. 16, 1391-1409 (2000).

3. Li, Z., Pradeep, K. G., Deng, Y., Raabe, D. \& Tasan, C. C. Metastable highentropy dual-phase alloys overcome the strength-ductility trade-off. Nature 534, 227-230 (2016).

4. Yeh, J. W. et al. Nanostructured high-entropy alloys with multiple principal elements: novel alloy design concepts and outcomes. Adv. Eng. Mater. 6, 299-303 (2004).

5. Cantor, B., Chang, I. T. H., Knight, P. \& Vincent, A. J. B. Microstructural development in equiatomic multicomponent alloys. Mater. Sci. Eng. A 375-377, 213-218 (2004).

6. Gludovatz, B. et al. A fracture-resistant high-entropy alloy for cryogenic applications. Science 345, 1153-1158 (2014).

7. Gludovatz, B. et al. Exceptional damage-tolerance of a medium-entropy alloy CrCoNi at cryogenic temperatures. Nat. Commun. 7, 10602 (2016).

8. Zhang, Y. et al. Microstructures and properties of high-entropy alloys. Prog. Mater. Sci. 61, 1-93 (2014).

9. Zhang, W., Liaw, P. K. \& Zhang, Y. Science and technology in high-entropy alloys. Sci. China Mater. 61, 2-22 (2018)

10. Zhang, Y. et al. Serration and noise behaviors in materials. Prog. Mater. Sci. 90, 358-460 (2017).

11. Miracle, D. B. \& Senkov, O. N. A critical review of high entropy alloys and related concepts. Acta Mater. 122, 448-511 (2017).

12. Diao, H. Y., Feng, R., Dahmen, K. A. \& Liaw, P. K. Fundamental deformation behavior in high-entropy alloys: an overview. Curr. Opin. Solid State Mater. Sci. 21, 252-266 (2017).

13. $\mathrm{Li}$, D. et al. High-entropy $\mathrm{Al}_{0.3} \mathrm{CoCrFeNi}$ alloy fibers with high tensile strength and ductility at ambient and cryogenic temperatures. Acta Mater. 123, 285-294 (2017).

14. Li, R. X., Liaw, P. K. \& Zhang, Y. Synthesis of AlxCoCrFeNi high-entropy alloys by high-gravity combustion from oxides. Mater. Sci. Eng. A 707, 668-673 (2017).

15. Luo, H., Li, Z. \& Raabe, D. Hydrogen enhances strength and ductility of an equiatomic high-entropy alloy. Sci. Rep. 7, 9892 (2017).

16. Li, Z., Tasan, C. C., Springer, H., Gault, B. \& Raabe, D. Interstitial atoms enable joint twinning and transformation induced plasticity in strong and ductile high-entropy alloys. Sci. Rep. 7, 40704 (2017).

17. Li, Z., Tasan, C. C., Pradeep, K. G. \& Raabe, D. A TRIP-assisted dual-phase high-entropy alloy: grain size and phase fraction effects on deformation behavior. Acta Mater. 131, 323-335 (2017).

18. Wang, M., Li, Z. \& Raabe, D. In-situ SEM observation of phase transformation and twinning mechanisms in an interstitial high-entropy alloy. Acta Mater. 147, 236-246 (2018).

19. Gao, M. C., Yeh, J. W., Liaw, P. K. \& Zhang, Y. High-entropy Alloys: Fundamentals and Applications (Springer, Switzerland, 2016).

20. Gali, A. \& George, E. P. Tensile properties of high- and medium-entropy alloys. Intermetallics 39, 74-78 (2013).

21. Deng, Y. et al. Design of a twinning-induced plasticity high entropy alloy. Acta Mater. 94, 124-133 (2015).

22. De Cooman, B. C., Chin, K. G. \& Kim, J. K. in New Trends and Developments in Automotive System Engineering (ed Chiaberge, M.) Ch. 6, 101-128 (InTech, 2011).

23. Rémy, L. \& Pineau, A. Twinning and strain-induced f.c.c. $\rightarrow$ h.c.p. transformation on the mechanical properties of $\mathrm{Co}-\mathrm{Ni}-\mathrm{Cr}-\mathrm{Mo}$ alloys. Mater. Sci. Eng. 26, 123-132 (1976).

24. Frommeyer, G., Brüx, U. \& Neumann, P. Supra-ductile and high-strength manganese-TRIP/TWIP steels for high energy absorption purposes. ISIJ Int. 43, 438-446 (2003).

25. Allain, S., Chateau, J. P., Bouaziz, O., Migot, S. \& Guelton, N. Correlations between the calculated stacking fault energy and the plasticity mechanisms in Fe-Mn-C alloys. Mater. Sci. Eng. A 387-389, 158-162 (2004).

26. Dumay, A., Chateau, J. P., Allain, S., Migot, S. \& Bouaziz, O. Influence of addition elements on the stacking-fault energy and mechanical properties of an austenitic Fe-Mn-C steel. Mater. Sci. Eng. A 483-484, 184-187 (2008).

27. Jo, M. et al. Theory for plasticity of face-centered cubic metals. Proc. Natl. Acad. Sci. U.S.A. 111, 6560-6565 (2014).

28. Li, B. Q., Sui, M. L. \& Mao, S. X. Twinnability predication for fcc metals. J. Mater. Sci. Technol. 27, 97-100 (2011).

29. Asaro, R. J. Suresh, S. Mechanistic models for the activation volume and rate sensitivity in metals with nanocrystalline grains and nano-scale twins. Acta Mater. 53, 3369-3382 (2005)

30. Ishida, K. Direct estimation of stacking fault energy by thermodynamic analysis. Phys. Status Solidi A 36, 717-728 (1976).

31. Li, W. et al. First-principles prediction of the deformation modes in austenitic Fe-Cr-Ni alloys. Appl. Phys. Lett. 108, 081903 (2016).

32. Tracy, C. L. et al. High pressure synthesis of a hexagonal close-packed phase of the high-entropy alloy CrMnFeCoNi. Nat. Commun. 8, 15634 (2017).
33. Zhang, F. et al. Polymorphism in a high-entropy alloy. Nat. Commun. 8 , 15687 (2017).

34. Zhao, S., Stocks, G. M. \& Zhang, Y. Stacking fault energies of face-centered cubic concentrated solid solution alloys. Acta Mater. 134, 334-345 (2017).

35. Ma, D., Grabowski, B., Körmann, F., Neugebauer, J. \& Raabe, D. Ab initio thermodynamics of the CoCrFeMnNi high entropy alloy: importance of entropy contributions beyond the configurational one. Acta Mater. 100, 90-97 (2015).

36. Zhang, Z. et al. Dislocation mechanisms and 3D twin architectures generate exceptional strength-ductility-toughness combination in CrCoNi mediumentropy alloy. Nat. Commun. 8, 14390 (2017).

37. Li, Z., Körmann, F., Grabowski, B., Neugebauer, J. \& Raabe, D. Ab initio assisted design of quinary dual-phase high-entropy alloys with transformation-induced plasticity. Acta Mater. 136, 262-270 (2017).

38. Huang, S. et al. Temperature dependent stacking fault energy of FeCrCoNiMn high entropy alloy. Scr. Mater. 108, 44-47 (2015).

39. Van Swygenhoven, H., Derlet, P. M. \& Froseth, A. G. Stacking fault energies and slip in nanocrystalline metals. Nat. Mater. 3, 399-403 (2004)

40. Zhang, F. X. et al. Pressure-induced fcc to hcp phase transition in Ni-based high entropy solid solution alloys. Appl. Phys. Lett. 110, 011902 (2017).

41. Miao, J. et al. The evolution of the deformation substructure in a $\mathrm{Ni}-\mathrm{Co}-\mathrm{Cr}$ equiatomic solid solution alloy. Acta Mater. 132, 35-48 (2017).

42. Li, W. et al. Generalized stacking fault energies of alloys. J. Phys. Condens. Matter 26, 265005 (2014).

43. Ogata, S., Li, J. \& Yip, S. Ideal pure shear strength of aluminum and copper. Science 298, 807 (2002).

44. Laplanche, G., Kostka, A., Horst, O. M., Eggeler, G. \& George, E. P. Microstructure evolution and critical stress for twinning in the $\mathrm{CrMnFeCoNi}$ high-entropy alloy. Acta Mater. 118, 152-163 (2016).

45. Ogata, S., Li, J., Hirosaki, N., Shibutani, Y. \& Yip, S. Ideal shear strain of metals and ceramics. Phys. Rev. B 70, 104104 (2004).

46. Olson, G. B. \& Cohen, M. A general mechanism of martensitic nucleation: part I. General concepts and the FCC $\rightarrow$ HCP transformation. Metall. Trans. A 7, 1897-1904 (1976).

47. Li, R. H. et al. Stacking fault energy of face-centered cubic metals: thermodynamic and ab initio approaches. J. Phys. Condens. Matter 28, 10 (2016).

48. Talonen, J. \& Hänninen, H. Formation of shear bands and strain-induced martensite during plastic deformation of metastable austenitic stainless steels. Acta Mater. 55, 6108-6118 (2007).

49. Kibey, S., Liu, J. B., Curtis, M. J., Johnson, D. D. \& Sehitoglu, H. Effect of nitrogen on generalized stacking fault energy and stacking fault widths in high nitrogen steels. Acta Mater. 54, 2991-3001 (2006).

50. Jo, M., Koo, Y. M. \& Kwon, S. K. Determination of the deformation mechanism of Fe-Mn alloys. Met. Mater. Int. 21, 227-231 (2015).

51. Jin, Z. H., Dunham, S. T., Gleiter, H., Hahn, H. \& Gumbsch, P. A universal scaling of planar fault energy barriers in face-centered cubic metals. Scr. Mater. 64, 605-608 (2011).

52. Li, W. et al. Generalized stacking fault energy of $\gamma$-Fe. Philos. Mag. 96, 524-541 (2016).

53. Ferreira, P. J. \& Müllner, P. A thermodynamic model for the stacking-fault energy. Acta Mater. 46, 4479-4484 (1998).

54. Perdew, J. P., Burke, K. \& Ernzerhof, M. Generalized gradient approximation made simple. Phys. Rev. Lett. 77, 3865-3868 (1996).

55. Vitos, L. Computational Quantum Mechanics for Materials Engineers (Springer, London, 2007).

56. Sato, K. et al. First-principles theory of dilute magnetic semiconductors. Rev. Mod. Phys. 82, 1633-1690 (2010).

57. Györffy, B. L., Pindor, A. J., Staunton, J., Stocks, G. M. \& Winter, H. A firstprinciples theory of ferromagnetic phase transitions in metals. J. Phys. F: Met. Phys. 15, 1337-1386 (1985).

\section{Acknowledgments}

This work was supported by the Swedish Research Council, the Swedish Foundation for Strategic Research, Sweden's Innovation Agency (VINNOVA Grant No. 2014-03374), the Swedish Foundation for International Cooperation in Research and Higher Education, the Carl Tryggers Foundation, the National Research Foundation of Korea (NRF) grant funded by the Korea government (MSIT) (NRF-2017R1A2A1A18071775), the China Scholarship Council, and the Hungarian Scientific Research Fund (OTKA 109570). The Swedish National Infrastructure for Computing at the National Supercomputer Centers in Linköping and Stockholm are acknowledged.

\section{Author contributions}

S.H., S.K.K., and L.V. initiated the research; S.H., W.L., and H.H. performed the calculations; S.H., H.H., D.K., S.L., X.L., E.H., S.K.K., and L.V. analyzed the data. S.H., S.K.K., 
and L.V. wrote the paper. All authors participated in the discussion and interpretation of the results.

\section{Additional information}

Supplementary Information accompanies this paper at https://doi.org/10.1038/s41467018-04780-x.

Competing interests: The authors declare no competing interests.

Reprints and permission information is available online at http://npg.nature.com/ reprintsandpermissions/

Publisher's note: Springer Nature remains neutral with regard to jurisdictional claims in published maps and institutional affiliations. (c) (i) Open Access This article is licensed under a Creative Commons Attribution 4.0 International License, which permits use, sharing, adaptation, distribution and reproduction in any medium or format, as long as you give appropriate credit to the original author(s) and the source, provide a link to the Creative Commons license, and indicate if changes were made. The images or other third party material in this article are included in the article's Creative Commons license, unless indicated otherwise in a credit line to the material. If material is not included in the article's Creative Commons license and your intended use is not permitted by statutory regulation or exceeds the permitted use, you will need to obtain permission directly from the copyright holder. To view a copy of this license, visit http://creativecommons.org/ licenses/by/4.0/.

(C) The Author(s) 2018 